\title{
Electron Confinement Effect of Laser Dyes within Dendritic Structures
}

\author{
Francisco Márquez ${ }^{1}$; María José Sabater ${ }^{2}$ \\ ${ }^{1}$ School of Science and Technology, Turabo University, 00778PR, USA. \\ ${ }^{2}$ Universidad Politécnica de Valencia-C.S.I.C., Av. Naranjos s/n, 46071-Valencia, Spain \\ E-mail address: fmarquez@suagm.edu \\ Received February 19, 2011; revised March 18, 2011; accepted March 25, 2011
}

\begin{abstract}
Dendrimers are a novel class of nanometric-size macromolecules with a regular tree-dimensional like array of branch units [1,2]. Their synthetic availability in a wide range of sizes combined with their peculiar architecture makes them versatile building blocks for a wide range of potential applications [3]. Some years ago, Meijer and co-workers reported that the modification of terminal amine functionalities of a fifth generation poly(propyleneimine) dendrimer (DAB-dendr- $\left.\left(\mathrm{NH}_{2}\right)_{64}\right)$ with bulky substituents, (typically N-t-BOC protected phenylalanine), results in the formation of the so-called "dendritic box" (DAB-dendr-(NH-tBOC-L-Phe $)_{64}$ [ [4]. Within this macromolecular structure it is possible to encapsulate a variety of guest molecules due to the existence of internal cavities in the core. The photophysical properties of the guests can be modulated by the innovative electron confinement effect. In this respect, we wish to report that the emission frequency of organic dyes can be easily modulated by encapsulation in a dendritic box. The emission bands of dye molecules incorporated into a dendrimer can effectively be red shifted with respect to their emission in solution and contrary to other confined spaces of considerable hardness, the magnitude of this shifting can be regulated under appropriate experimental conditions. This peculiar effect could have unprecedented applications in the development of supramolecular devices relating to the frequency tuning of organic laser dyes.
\end{abstract}

Keywords: Dendrimer, Confinement, Dyes

\section{Introduction}

The electron confinement effect, that has been experimentally observed in organic guests included within zeolite hosts, is based on the concept that the molecular orbitals of the adsorbates inside the host cavities are not extended over all the space, as they are in the gas phase, but instead are forced to limit within a reduced and regular space [5]. This confinement effect is stronger as the size of the confined guest approaches the cavity dimensions, producing an energy increase of all molecular orbitals [6]. The HOMO has been predicted to be more sensitive that the LUMO, and the predicted effect is a reduction of the HOMO-LUMO energy gap that is reflected in the optical properties of the guest, particularly the bathochromic shift of the fluorescence emission [6].

In principle, the polar or non-polar nature of the dendritic shell can establish different interactions with solvents and this property is expected to alter the size of the dendritic box in a specific way. Thus, we envisaged that a chemical consequence of this is that solvation effects could lead to either an enlargement or a reduction of the original macromolecular dimensions by changing the solvent polarity. This would leave no alternative but to temporarily deform the size of the dendritic cavities inside. As a consequence, guest molecules incorporated within the dendritic cavities might interact differently with the dendritic walls influenced by the solvent environment. When the dendritic cavity and the guest dimensions are closer, the interactions can be sufficiently important as to experience strong repulsions. Such should be the case when solvents of opposite polarity to the dendritic system are employed. This stronger interaction may modify the HOMO-LUMO energy gap of the guest resulting in appreciable modifications of the photophysical properties of the guest. This concept, which has been documentarily confirmed by theoretical predictions and recent experimental results, is a reflection of the con- 
finement effect imposed by the host [5,6].

In order to test the validity of these assumptions, we studied the photophysics of several dye molecules incorporated into a "dendritic box" of a fifth generation poly (propyleneimine) dendrimer (DAB-dendr-(NH-tBOC-L-Phe $)_{64}$ ). These experiments were conducted in solvents of pronounced different polarity and the photophysical data were compared to those of dye molecules in neat solvents.

\section{Experimental}

For the investigation presented here, three dye molecules (Bengal Rose 1, Rhodamine B 2 and Congo Red 3) were encapsulated in three different dendritic boxes constructed from a fifth generation poly (propylene imine) dendrimer with 64 terminal amine groups [DABdendr-(NH-t-BOC-L-Phe) ${ }_{64}$ ] and a L-phenylalanine derivative.

The dendritic box was synthesized according to a previously reported procedure [4b]. Hence, in a typical procedure $36 \mathrm{mg}$ of Rose Bengal $\left(3.5 \times 10^{-5} \mathrm{~mol}\right)$ or $24.5 \mathrm{mg}$ of Red Congo $\left(3.5 \times 10^{-5} \mathrm{~mol}\right)$ and $16.9 \mathrm{mg}$ of Rhodamine $\mathrm{B}\left(3.5 \times 10^{-5} \mathrm{~mol}\right)$ was added to a solution of $50 \mathrm{mg}$ of dendrimer DAB-dendr-(NH-t-BOC-L-Phe)64 (64 end amine groups) in $5 \mathrm{~mL}$ of $\mathrm{CH}_{2} \mathrm{Cl}_{2}$ with $1 \mathrm{~mL}$ of trie- thylamine and the solutions were stirred for $24 \mathrm{~h}$ at room temperature. Then $161 \mathrm{mg}$ of N-tBOC-L-phenylalanine $\mathrm{N}$-hydroxy-succinimide ester was added and the solutions were stirred overnight. Dilution to approx. 50ml with dichloromethane was followed by washing with water and saturated $\mathrm{Na}_{2} \mathrm{CO}_{3}$ solution. The organic layer was dried with $\mathrm{MgSO}_{4}$ and the solvent was evaporated under vacuum to afford the dye-doped dendrimer. In cases where the dyes were poorly soluble in water, the dendrimer was purified by dialysis until no traces of free dye were detected by HPLC.

Dye-doped dendrimers were characterized by matrix-assisted laser desorption ionisation time-of-flight mass spectroscopy (MALDI-TOF-MS), ${ }^{1} \mathrm{H}$ and ${ }^{13} \mathrm{C}$ nuclear magnetic resonance (NMR), ultraviolet and infrared spectroscopy and by cryo-Scanning Electron Microscopy.

\section{Results and Discussion}

The concentration of encapsulated guests was estimated by elemental analysis by determining the chlorine and sulfur content for compounds $\mathbf{1}$ and $\mathbf{2}$ respectively. The loading of compound $\mathbf{3}$ was estimated by UV/vis spectroscopy. In all cases, the loading level of the dye incorporated into the dendritic structure was ca. 3 dye molecules per dendrimer.
The excitation and emission spectra of pure 1 in ethanol were recorded at room temperature. The emission spectrum consisted of a sharp and featureless band around $574 \mathrm{~nm}$ and a small shoulder at ca. $620 \mathrm{~nm}$. Similar spectra could be obtained when the spectra were registered in the less polar solvents dichloromethane and n-hexane (see Figure 1).

Interestingly, when the same dye molecule was encapsulated within the dendrimer, a progressive red shifting of the band could be measured when the medium polarity was reduced (from $8 \mathrm{~nm}$ in dichloromethane to 37 $\mathrm{nm}$ in n-hexane) (see Figure 2a).

As for 1, common organic solvents such as ethanol, dichloromethane and n-hexane exerted hardly any noticeable influence on the emission spectra of pure dyes 2 and 3. Nevertheless, and as expected, the emission spectra of $\mathbf{2}$ and $\mathbf{3}$ when incorporated into the dendritic box underwent a remarkable red shifting (see Figure 2 b-c). The fluorescence band of the dye $\mathbf{2}$ incorporated into the dendritic structure displayed a maximum bathocromic shift of ca. $30 \mathrm{~nm}$. However, the most spectacular red shift was observed in the case of the dye 3-doped dendrimer in which a red shift of ca. $200 \mathrm{~nm}$ was measured in n-hexane with respect to the emission spectrum recorded in ethanol.

The loading level of the dyes incorporated into the dendritic structures was similar in all cases and for this reason an explanation to these experimental results should be found in the increase of the spatial restrictions imposed to the dye, leading to an enhancement of the observed electron confinement effect. To rationalize these results it is necessary to consider that non polar solvents such as n-hexane can cause a dramatic shrinkage of the original size of the macromolecule. In addition, the non-polar character of the solvent may induce a much closer association of the dye-doped dendrimers or even its aggregation (Figure 3 displays a simulation of the dye 1-doped dendrimer indicating the possible equilibrium in polar and non-polar solvents). The combination of both phenomenons can paradigmatically lead to an effective reduction of the internal dendritic cavities where the guest molecules are located, hence inducing changes in the frontier orbitals of the dyes to an extent that will depend on the degree of interaction exerted by the branches of the macromolecule. This stronger confinement is reflected by the notable reduction of the energy gap $\mathrm{S} 0 \rightarrow \mathrm{S} 1 *$ and consequently by a bathocromic shift of the emission spectra of the guests. Obviously, this effect is stronger when a host-guest tight fit occurs (a requirement that can be achieved when non-polar solvents as n-hexane are used).

Finally, the room temperature fluorescence lifetimes for the dyes incorporated into the dendritic structures 


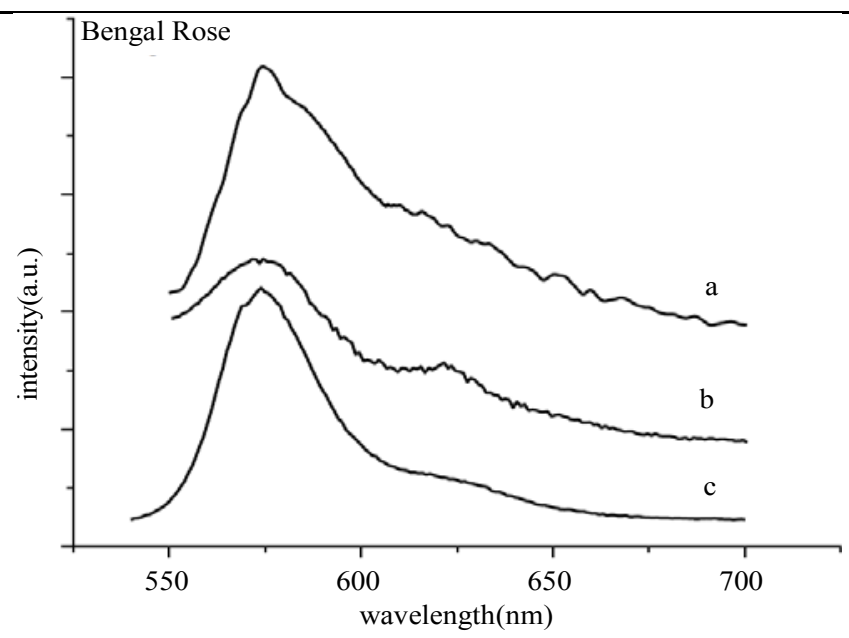

Figure 1. Emission spectra at room temperature of Bengal Rose in different solvents: (a) Ethanol, (b) Dichloromethane and (c) Pentane.

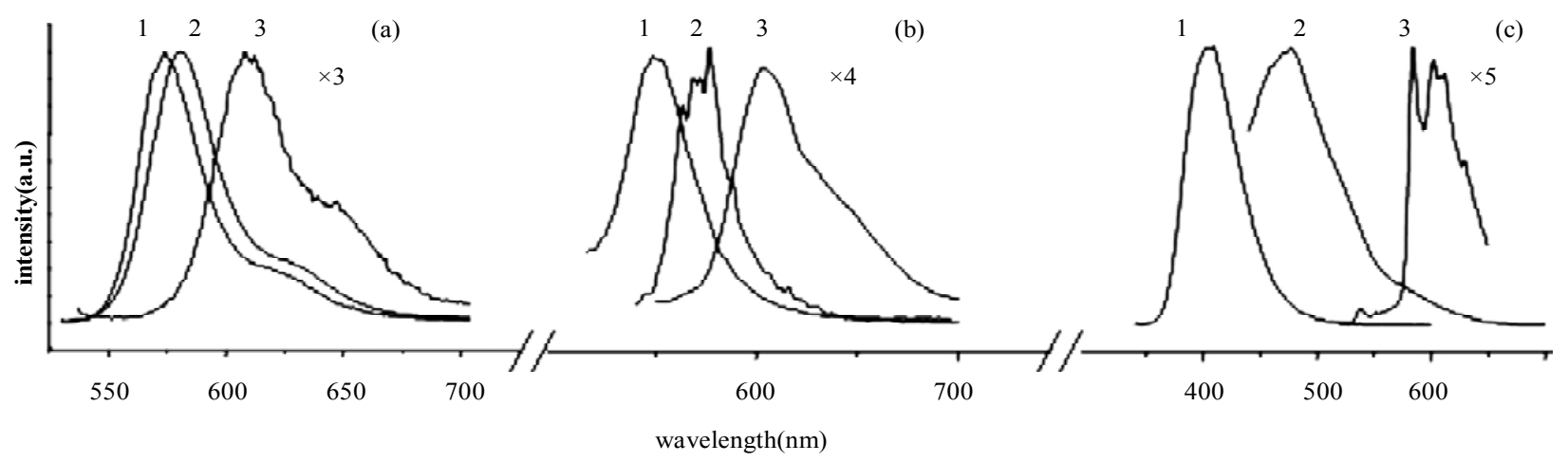

Figure 2. Emission spectra at room temperature of Bengal Rose (a), Rhodamine B (b), and Congo Red (c) included within the dendritic structure in different solvents: (1) Ethanol, (2) Dichloromethane and (3) N-hexane.
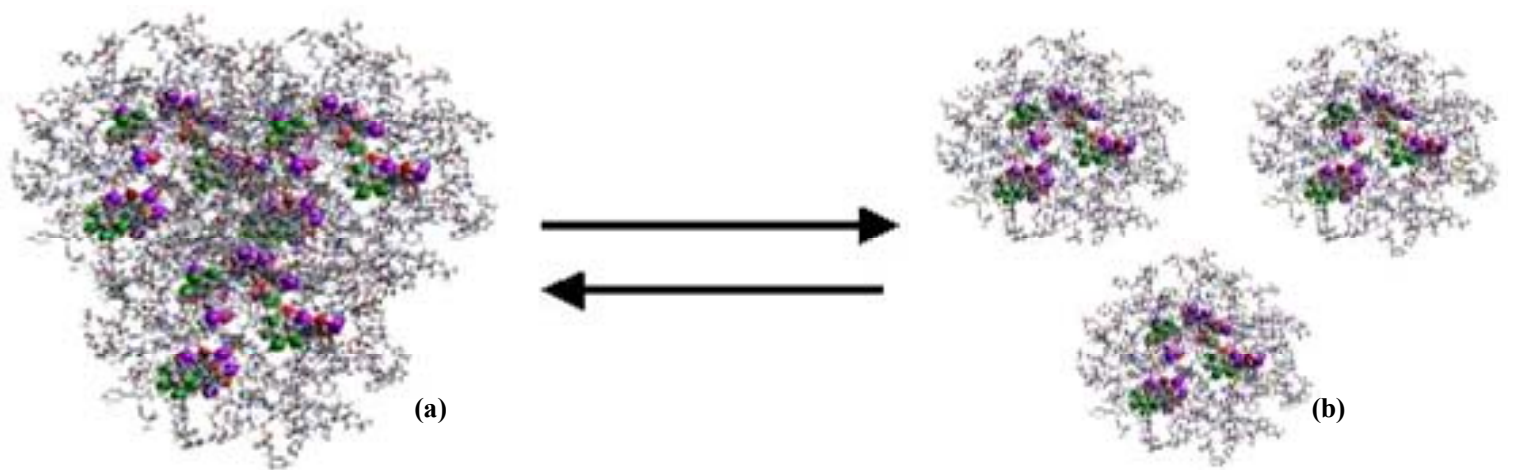

Figure 3. Theoretical Simulation of the equilibrium experimented by three dendritic structures with three molecules of Bengal Rose incorporated within the structure, in non polar (a) and polar (b) solvent.

were adjusted to single-exponential functions. Figure $\mathbf{4}$ shows the fluorescence decays obtained for RhodamineB@dendrimer in different solvents. These constants, which were lower than the ones measured for the pure dye in ethanol $(\tau=5.1 \mathrm{~ns})$ decreased substantially from ethanol (3.1 ns) to dichloromethane $(1.1 \mathrm{~ns})$ and $\mathrm{n}$-hexane $(0.5 \mathrm{~ns})$. This kinetic behaviour resulting from the orbital-confinement effect enhances the non radiative 


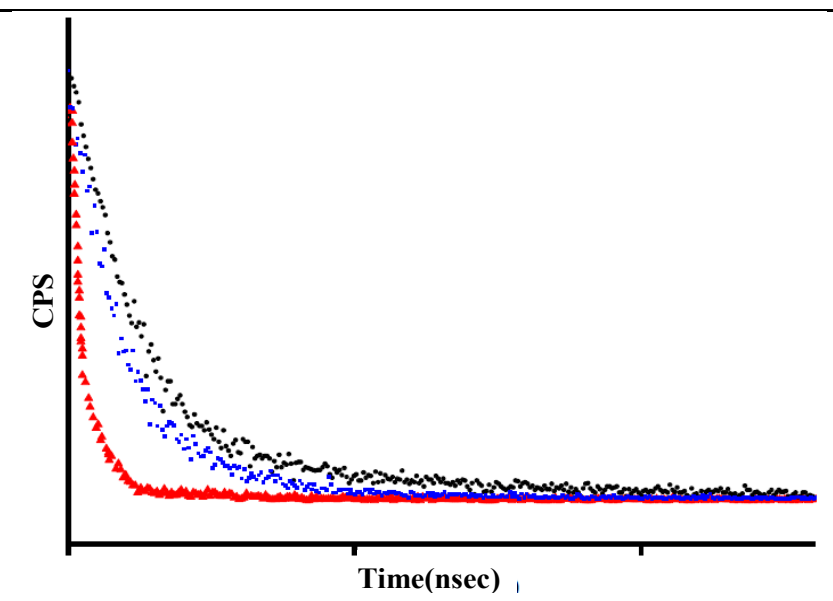

Figure 4. Normalized fluorescence decays of RhodamineB@dendrimer in different solvents: (•) Ethanol, (•) Dichloromethane and $(\Delta) \mathrm{N}$-Hexane, after excitation at the maximum absorption wavelength.

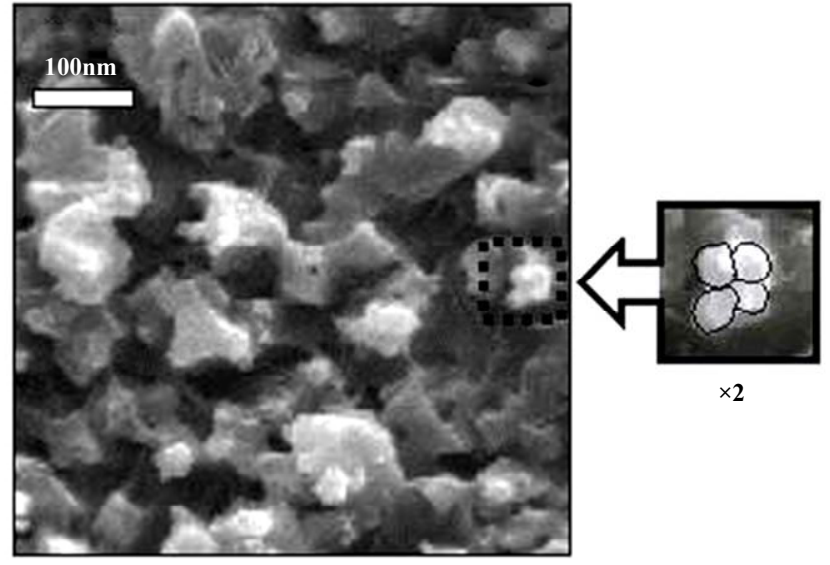

(a)

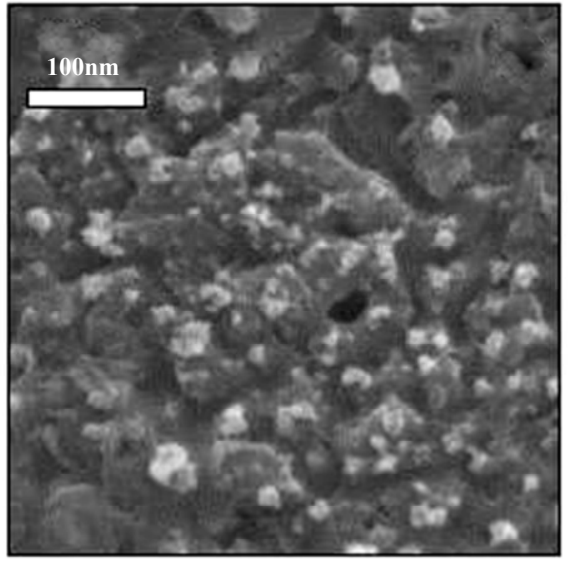

(b)

Figure 5. Cryo-Scanning electron microscopy of a sample of doped dendrimer (Bengal Rose@dendrimer) in n-hexane (a) and ethanol (b). From this figure can be derived the different aggregation state depending on the solvent polarity.

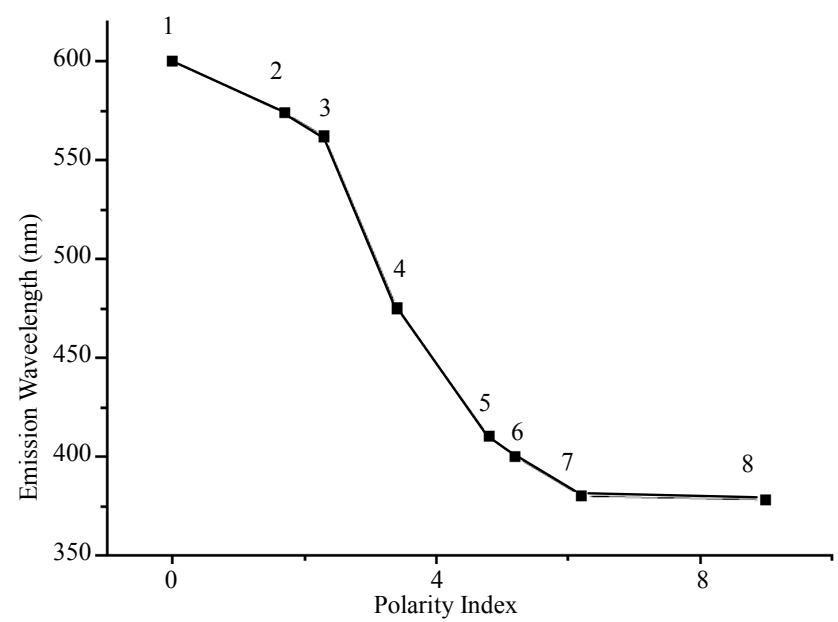

Figure 6. Fluorescence emission of Congo Red@dendrimer in different solvents: n-hexane (1), carbon tetrachloride (2), toluene (3), dichloromethane (4), 1-4 dioxane (5), ethanol (6), acetonitrile (7), water (8). 
deactivation pathway due to the closer proximity between the lowest excited singlet states.

Experimental evidence of this dynamic equilibrium observed in dendrimers exposed to different polarity environments has been obtained from cryo-SEM (cryo- scanning electron microscopy). Cryo-SEM was the method of choice for investigating the dendritic structures in different solvents because the samples were in solution. This technique can be a particularly useful method for studying new types and interaction modes among solvents and dendrimers. This study was performed on the dye 1-doped dendrimer in ethanol and $n$-hexane. Samples were cooled to $-170^{\circ} \mathrm{C}$ and subsequently analysed (see Supplementary Information for experimental details). Figure 5 displays the results obtained by cryo-SEM indicating that increasing the polarity (in going from n-hexane to ethanol) substantially modifies both the molecular dimension and aggregation state of the doped dendrimers.

Other confined spaces that can affect the guest-host relationships similarly, in this case through the hardness of their walls, are the zeolite framework [6]. In fact, it has been reported that the photophysical properties of several probe molecules such as naphthalene or anthracene within pure silica zeolites are strongly affected by the zeolite host [6]. The magnitude of this shifting appears in theory to be lower than the one attained in this case for our dye-doped dendrimer. Even more, the absorption of a guest included in a dendritic box does not involve the disadvantage of light scattering typical of inorganic opaque solids, thus leading to much more efficient photochemical and photophysical processes.

To sum up, the ability of the dendrimers to create confined microenvironments in determined experimental conditions can be taken as an easy way to modify the emission spectrum of dye molecules trapped in their cavities. This may have important implications from the technological point of view since dye-doped dendrimers can serve to enlarge the frequency range of laser dyes simply by choosing a solvent or a mixture of solvents of suitable polarity. What is more, the ability of these dye-doped dendrimers to modify the emission frequency range simply by changing the medium polarity may have interesting applications in the development of new supramolecular devices. This is clearly exemplified in Figure 6, where the fluorescence emission wavelength of the dye 3-doped dendrimer in different solvents is plotted. As can be seen there, the fluorescence emission is clearly correlated with the polarity of the solvents and this effect may only be justified as due to the confinement effect of the dye.

\section{Acknowledgements}

Copyright (C) 2011 SciRes.
Generous financial support from NASA (Grant NAG10335 ) is gratefully acknowledged. Financial support from US Department of Energy through the Massey Chair project at University of Turabo is also acknowledged.

\section{References}

[1] J. M. J. Fréchet, "Functional Polymers and Dendrimers: Reactivity, Molecular Architecture, and Interfacial Energy," Science, Vol. 263, 1994, pp. 1710-1715. doi:10.1126/science.8134834

[2] G. R. Newkome, C. N. Moorefield and F. Vögtle, "Dendritic Molecules: Concepts, Synthesis, Perspectives; VCH: Weinheim, Germany, 1996.

A. W. Bosman, H. M. Janssen and E. W. Meijer, "About Dendrimers: Structure, Physical Properties, and Applications," Chemical Reviews, Vol. 99, 1999, pp. 1665-1688. doi:10.1021/cr970069y

D. A. Tomalia, "Starburst/Cascade Dendrimers: Fundamental Building Blocks for a New Nanoscopic Chemistry Set," Advanced Materials, Vol. 6, 1994, p. 529. doi:10.1002/adma.19940060703

H. F. Chow, T. K. K. Mong, M. F. Nongrum and C. W. Wan, "The Synthesis and Properties of Novel Functional Dendritic Molecules," Tetrahedron, Vol. 54, 1998, pp. 8543-8660. doi:10.1016/S0040-4020(98)00409-8

S. M. Grayson and J. M. J. Fréchet, "Convergent Dendrons and Dendrimers: From Synthesis to Applications," Chemical Reviews, Vol. 101, 2001, pp. 3819-3868. doi:10.1021/cr990116h

[3] O. A. Matthews, A. N. Shipway and J. F. Stoddart, "Dendrimers-Branching out from Curiosities into New Technologies," Progress in Polymer Science, Vol. 23, No. 1, 1998, pp. 1-58. doi:10.1016/S0079-6700(97)00025-7

M. Fisher and F. Vögtle, "Dendrimers: From Design to Application-A Progress Report," Angewandte Chemie International Edition, Vol. 38, No. 7, 1999, pp. 884-905. doi:10.1002/(SICI)1521-3773(19990401)38:7<884::AIDANIE884>3.0.CO;2-K

S. Hecht and J. M. J. Fréchet, "Dendritic Encapsulation of Function: Applying Nature's Site Isolation Principle from Biomimetics to Materials Science," Angewandte Chemie International Edition, Vol. 40, No. 1, 2001, pp. 74-91.

doi:10.1002/1521-3773(20010105)40:1<74::AID-ANIE7 4>3.0.CO;2-C

D. Astruc and F. Chardac, "Dendritic Catalysts and Dendrimers in Catalysis," Chemical Reviews, Vol. 101, No. 9, 2001, pp. 2991-3024. doi:10.1021/cr010323t

[4] E. M. M. de Brabander-van der Berg and E. W. Meijer, "Poly (propylene imine) Dendrimers: Large-Scale Synthesis by Hetereogeneously Catalyzed Hydrogenations," Angewandte Chemie International Edition, Vol. 32, No. 9, 1993, pp. 1308-1311. doi:10.1002/anie.199313081

J. F. G. A. Jansen, E. M. M. de Brabander-van der Berg and E. W. Meijer, "Encapsulation of Guest Molecules into a Dendritic Box," Science, Vol. 266, No. 5188, 1994, pp. 1226-1229. doi:10.1126/science.266.5188.1226

[5] C. M. Zicovich-Wilson, P. Viruela and A. Corma, "Elec- 
tronic Confinement of Molecules in Microscopic Pores. A New Concept Which Contributes to the Explanation of the Catalytic Activity of Zeolites," Journal of Physical Chemistry, Vol. 98, No. 42, 1994, pp. 10863-10870. doi:10.1021/j100093a030

[6] F. Márquez, C. M. Zicovich-Wilson, A. Corma, E. Palomares, H. García, "Naphthalene Included within All-Silica Zeolites: Influence of the Host on the Naphthalene Photophysics," Journal of Physical Chemistry, Vol. 105, No. 4, 2001, pp. 9973-9979. doi:10.1021/jp012095c

F. Márquez, H. García, E. Palomares, A. Corma and L.

Fernandez, "Spectroscopic Evidence in Support of the
Molecular Orbital Confinement Concept: Case of Anthracene Incorporated in Zeolites," Journal of American Chemical Society, Vol. 122, No. 27, 2000, pp. 6520-6521. doi:10.1021/ja0003066

F. Márquez, V. Martí, E. Palomares, A. García and W. Adam, Journal of American Chemical Society, Vol. 124, 2002, p. 7264

B. Menaa, M. Takahashi, Y. Tokuda and T. Yoko, Journal of Photochemistry and Photobiology A: Chemistry, Vol. 194, 2008, p. 362. 